\title{
An Ongoing Mid-infrared Outburst in the White Dwarf 0145+234: Catching in Action the Tidal Disruption of an Exoasteroid?
}

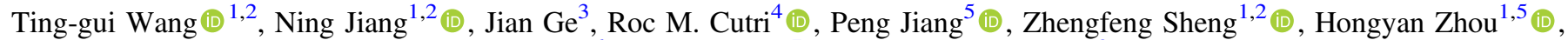 \\ James Bauer ${ }^{6}$, Amy Mainzer ${ }^{7}$, and Edward L. Wright ${ }^{8}$ (i) \\ ${ }^{1}$ CAS Key Laboratory for Researches in Galaxies and Cosmology, University of Sciences and Technology of China, Hefei, Anhui 230026, People's Republic of \\ China; twang@ustc.edu.cn \\ ${ }^{2}$ School of Astronomy and Space Sciences, University of Science and Technology of China, Hefei, 230026, People's Republic of China \\ ${ }^{3} 211$ Bryant Space Science Center, Department of Astronomy, University of Florida, Gainesville, FL 32611-2055, USA \\ ${ }^{4}$ IPAC/Caltech, 1200 E. California Boulevard, Pasadena, CA 91125, USA \\ 5 Antarctic Astronomy Research Division, Key Laboratory for Polar Science of the State Oceanic Administration, Polar Research Institute of China, Shanghai, \\ People's Republic of China \\ ${ }^{6}$ Department of Astronomy, University of Maryland, College Park, MD 20742, USA \\ ${ }^{7}$ University of Arizona, 1629 E. University Boulevard, Tucson, AZ 85721, USA \\ ${ }^{8}$ Division of Astronomy and Astrophysics, University of California, Los Angeles, Los Angeles, CA 90095, USA \\ Received 2019 September 17; revised 2019 November 2; accepted 2019 November 4; published 2019 November 14
}

\begin{abstract}
We report the detection of a large-amplitude MIR outburst in the white dwarf (WD) $0145+234$ in the NEOWISE Survey data. The source had a stable MIR flux before 2018, and was brightened by about 1.0 magnitude in the $W 1$ and $W 2$ bands within half a year and has been continuously brightening since then. No significant variations are found in the optical photometry data during the same period. This suggests that this MIR outburst is caused by recent replenishing or redistribution of dust, rather than intrinsic variations of the WD. Spectral energy distribution modeling of $0145+234$ suggests that there was already a dust disk around the WD in the quiescent state, and both of the temperature and surface area of the disk evolved rapidly since the outburst. The dust temperature was $\simeq 1770 \mathrm{~K}$ in the initial rising phase, close to the sublimation temperature of silicate grains, and gradually cooled down to around $1150 \mathrm{~K}$, while the surface area increased by a factor of about six during the same period. The inferred closest distance of dust to the WD is within the tidal disruption radius of a gravitationally bounded asteroid. We estimated the dust mass to be between $3 \times 10^{15}$ and $3 \times 10^{17} \rho /\left(1 \mathrm{~g} \mathrm{~cm}^{-3}\right) \mathrm{kg}$ for silicate grains of a power-law size distribution with a high cutoff size from 0.1 to $1000 \mu \mathrm{m}$. We interpret this as a possible tidal breakup of an exoasteroid by the WD. Further follow-up observations of this rare event may provide insights on the origin of dust disk and metal pollution in some WDs.
\end{abstract}

Unified Astronomy Thesaurus concepts: White dwarf stars (1799); Circumstellar dust (236); Infrared excess (788); Variable radiation sources (1759)

\section{Introduction}

A substantial fraction of white dwarfs (WDs) are known to show metal absorption lines in their spectra (Zuckerman et al. 2010; Koester et al. 2014). Because heavy elements diffuse out of the photosphere in a rather short time (days to hundred years, depending on $T_{\text {eff }}$ and $\log g$ ) in the strong surface gravity of a WD 9 (Koester 2009; Bergeron et al. 2015), this indicates that these heavy elements were added to the surface very recently, presumably, by accretion of tidally disrupted asteroids or comets (Jura \& Young 2014). Many WDs also display infrared excesses, which may be considered as evidence for dust disks (Zuckerman \& Becklin 1987; Farihi et al. 2009; Rebassa-Mansergas et al. 2019). The presence of a warm disk is further supported by the detection of doublepeaked infrared Ca II emission lines (Jura 2003; Gänsicke et al. 2006; Veras et al. 2014). Dust in the disk in a WD is either transient or continuously replenished because grains can be dragged onto the star through the Poynting-Robertson force on timescales of several years (e.g., Reach et al. 2005; Rafikov 2011). Fragmentation of a planetary body has been proposed as a source of dust (Jura \& Young 2014; Vanderburg

\footnotetext{
9 http://montrealwhitedwarfdatabase.org/evolution.html
}

et al. 2015; Manser et al. 2019). Variability in infrared flux on a timescale from less than a year to 10 years has been reported very recently in a number of WDs (Farihi et al. 2018; Xu et al. 2018; Swan et al. 2019a), but most of variations are generally of small amplitudes (e.g., 20\%-40\%, Xu \& Jura 2014; Xu et al. 2018; Swan et al. 2019b) on timescales of a year.

In this Letter, we report the discovery of a large MIR outburst in the WD 0145+234 (01:47:54.81 + 23:39:43.6; Wills \& Wills 1974). This outburst has so far lasted one and a half years. This event was discovered during a blind search for large-amplitude MIR outbursts in the WISE/NEOWISE data archive (Wright et al. 2010; Mainzer et al. 2014). This could be a process of tidal disruption caught in action.

\section{Data and Analysis}

The Wide-field Infrared Survey Explorer (WISE) all-sky survey and NEOWISE Reactivation mission conducted repeated scans of the entire sky at 3.4 and $4.6 \mu \mathrm{m}$ (hereafter Wl and W2) beginning in 2010 January through the present, except for the period 2011 February-2013 November. We retrieved the $W 1$ and $W 2$ point-spread function profile-fit photometry of $0145+234$ from the AllWISE Multi-epoch Photometry Table and NEOWISE-R Single Exposure (L1b) 


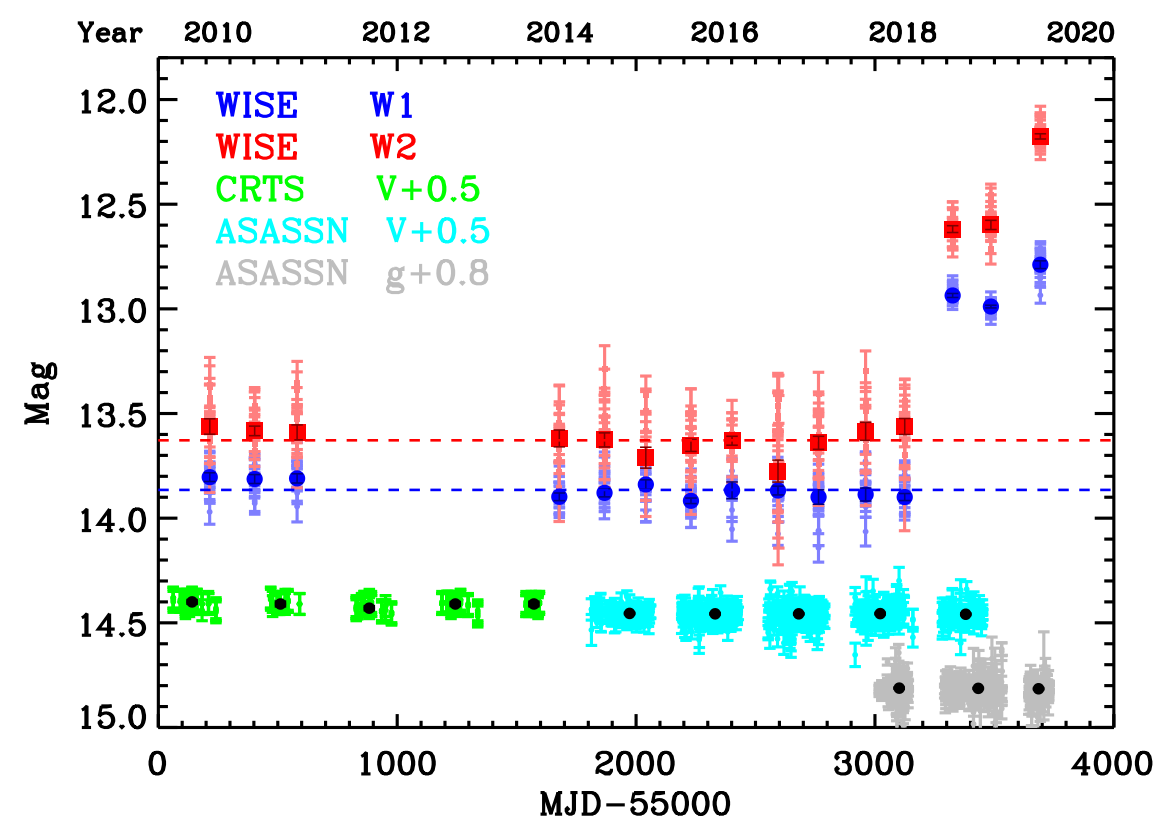

Figure 1. WISE/NEOWISE(-R) and optical light curves of the white dwarf $0145+234$. Legend: blue circles and red squares are the epoch medians of W1 and W2 magnitude. Black circles are median magnitudes in $V$ or $g$ over one observational season.

Source Table, ${ }^{10}$ which contain all measurements from 2010 to 2019 July. The single-exposure data were first screened by the quality flag marked in the catalogs to remove measurements with poor quality or possible corruption: (qual_frame $<5$ ), charged particle hits $\left(s a a_{-} s e p<5\right)$, scattered moon light (moon_masked $=1)$, and artifacts $\left(c c \_\right.$flags $\left.>0\right)$. The highquality measurements in each six-month observation epoch were averaged to increase the signal-to-noise ratio of the photometry, resulting in 15 epochs (see Figure 1). No significant variations are present in the first 12 epochs and thus we take them as the quiescent state with average magnitudes of $13.87 \pm 0.04$ and $13.63 \pm 0.06$ in the Wl and W2 bands, respectively. The light curve displays a large outburst between the 2018 January and 2018 July epochs $(\Delta W 1=0.93 \pm 0.04, \Delta W 2=1.01 \pm 0.06)$ and continues rising in the later epochs. Overall, the outburst appears to display a redder-when-brighter trend in which the variation amplitude in the $W 2$ band is larger than that in the WI band.

The brightening of $0145+234$ is not the result of object's motion carrying it close to a bright nearby source. The proper motion of the WD is measured by Gaia to be $\mu_{\text {R.A. }}=-5.21 \pm$ 0.12 and $\mu_{\text {decl. }}=-97.59 \pm 0.08$ mas year $^{-1}$ (Gaia Collaboration et al. 2018). Examination of the AllWISE Atlas Image (epoch 2010) shows that the closest MIR source is approximately $14^{\prime \prime}$ to the southwest of the WD position. The nearby source was cleanly separated and measured in the AllWISE Catalog, and is $1.6 \mathrm{mag}$ fainter in $W 1$ and $W 2$ than J0145+234 in 2010. Even if the WD moved directly onto that source, which it did not (see Figures 2 and 3), the apparent flux increase would be much too small to account for the brightening observed in the light curve.

The relative motion of $0145+234$ is clearly detected from the AllWISE and NEOWISE astrometry that spans a time baseline of $9 \mathrm{yr}$ and is fully consistent with proper motion measured by Gaia, as illustrated in Figure 4. The position of the white dwarf during outburst does not show any deviation

\footnotetext{
$\overline{10}$ https://irsa.ipac.caltech.edu/cgi-bin/Gator/nph-scan? mission=irsa\&submit=Select\&projshort=WISE
}

from the expected motion larger than $0 . \prime 1$. Thus, a chance coincidence with a bright nearby source could not have shifted the photocenter of the WD, making this occurrence unlikely.

$W 1$ and $W 2$ images comparing the region around $0145+234$ in 2014 January, 2018 January, and 2019 January are shown in Figures 2 and 3. The 2014 January and 2018 images are preoutburst, and the difference images between the 2018 and 2014 show only a very small positive/negative residual due to the slight motion of the source in the four years separating the observations. The differences between the 2019 and 2014 images show a bright, pointlike image at the position of the WD that is the source in outburst.

$0145+234$ is bright in the optical band $(V \sim 13.93 \mathrm{mag})$ and thus has been well measured by various optical time-domain surveys. We retrieved the optical photometry data from the publicly released data archive from the Catalina Real-time Transient Survey ${ }^{11}$ (CRTS; Drake et al. 2009) and All-Sky Automated Survey for Supernovae (ASAS-SN; Shappee et al. 2014; Kochanek et al. 2017). ${ }^{12}$ The CRTS survey has monitored $0145+234$ since 2005 without filters, but the photometry is calibrated to a pseudo- $V$ magnitude using a few dozen preselected standard stars in each field. The public CRTS data are available to 2013 October. Fortunately, the public ASAS-SN survey can serve the subsequent $V$-band photometry. Although the latest observations are performed in the $g$ band, these $g$-band data largely overlap with $V$-band data. In contrast to the remarkable MIR variability, $0145+234$ is quite stable and shows negligible variability in the long-term (more than one decade) optical light curves, including the MIR outburst period.

We made the quiescent spectral energy distribution (SED) of the WD by collecting data from GALEX, PANSTARRs (Chambers et al. 2016), Gaia (Gaia Collaboration et al. 2016), 2MASS (Skrutskie et al. 2006), and ALLWISE. We matched the UV to near-infrared photometry with the synthesized WD SED

\footnotetext{
${ }^{11}$ http://nunuku.caltech.edu/cgi-bin/getcssconedb_release_img.cgi

12 https://asas-sn.osu.edu/
} 


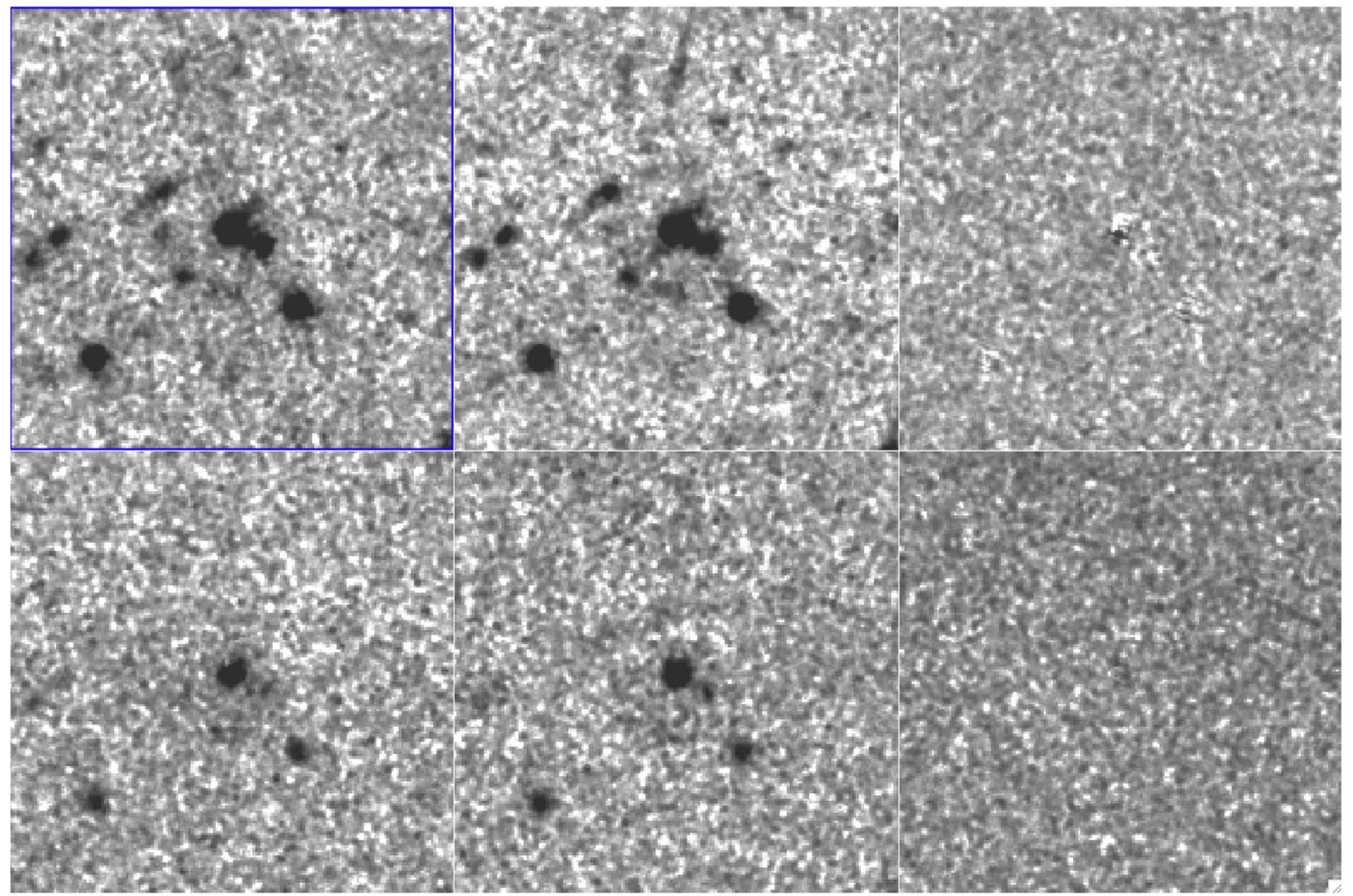

Figure 2. Top panels: montage of W1 images showing a $180^{\prime \prime} \times 180^{\prime \prime}$ region centered on the position of $0145+234$ from the AllWISE Catalog. The left panel shows the coaddition of the 2014 January single exposures, the center panel is the coaddition of the 2018 January single exposures, and the right panel is the difference between the 2018 and 2014 January images. Bottom panels: same as the top panels, but for $W 2$.

models (Figure 5). ${ }^{13}$ The models cover the range of $T_{\text {eff }}$ from $2500 \mathrm{~K}$ to $90,000 \mathrm{~K}$ and $\log g$ from 7.0 to 9.0 with a step of 0.5 for DA WDs, and $T_{\text {eff }}$ from $3250 \mathrm{~K}$ to $70,000 \mathrm{~K}$ and $\log g$ from 7.0 to 9.0 for DB WDs (Bergeron et al. 2011; Tremblay et al. 2011; Blouin et al. 2018). The interstellar reddening of the CCM law with $R_{V}=3.1$ (Cardelli et al. 1989) was added with the $E(B-V)$ as a free parameter. The best-fitted parameters converge to a model $T_{\text {eff }}=13,000 \mathrm{~K}, \log g=8.0$, and $E$ $(B-V)=0.014 \pm 0.004$. They are consistent with those derived from the spectroscopic model $(T=13,060 \pm 217 \mathrm{~K}$, $\log g=8.13 \pm 0.05$, Gianninas et al. 2011; $T=12,910 \pm$ $219 \mathrm{~K}, \log g=8.11 \pm 0.05$, Limoges et al. 2015). There is no evidence for a stellar companion for this WD either from broadband SEDs (Figure 5) or the optical spectrum. We examined their optical spectrum in the Montreal WD database, ${ }^{14}$ and found an absorption line of Ca II 3934. Unfortunately, Ca II 3969 is difficult to identify because it falls in the absorption trough of a Balmer absorption line. This suggests it is a DAZ WD. With these photospheric parameters, we also derived other parameters of the $\mathrm{WD}^{15}$ (Fontaine et al. 2001; Gianninas et al. 2011): the mass of $M_{*}=0.667 M_{\odot}$, the radius of $R_{\mathrm{WD}}=0.0116 R_{\odot}$, the luminosity of $L_{\mathrm{WD}}=0.00350$ $L_{\odot}$, and the age of $0.381 \mathrm{Gyr}$.

\footnotetext{
13 http://www.astro.umontreal.ca/ bergeron/CoolingModels

14 http://montrealwhitedwarfdatabase.org/WDs/WD $\% 200145+234 /$ WD $\%$ 200145+234.html

15 http://montrealwhitedwarfdatabase.org/evolution.html
}

\subsection{IR Excess in the Low State}

The observed ALLWISE fluxes in the W1,W2, and W3 $(12 \mu \mathrm{m})$ bands are clearly above the predicted values from the WD model (Figure 5). The excess over WD photospheric emission are significant at about $10 \sigma$ in the $W 1$ and $W 2$ bands, and $9 \sigma$ in the $W 3$ band. The infrared excess indicates a dust disk. Initially, we added a blackbody curve to model the infrared excess, and found that it is insufficient to fit all the data with a $2 \sigma$ excess in the $W 3$ band, which requires an additional temperature component or the non-gray dust model to fit. However, since a two-component model needs a total of four parameters (two temperatures and two surface areas), while only three data points $(W 1, W 2$, and $W 3)$ are available, such a model cannot be fully constrained. To illustrate the possibility of non-gray dust model, we adopt the particle-cluster-aggregation (PCA) model for olivine (Nakamura 1998), which is used to explain the scattering disk of $\beta$ Pic. Without increasing the number of free parameters, the model can now fit the data (Figure 5).

\subsection{Dust in the High State}

In the high state, this source is more than one magnitude brighter in the $W 1$ and $W 2$ bands. Since we only have two data points, we chose to fit the MIR excess with a singletemperature blackbody model. We considered two different scenarios: (1) the quiescent dust disk has been transformed into the latter one; (2) the quiescent disk remains the same and there 


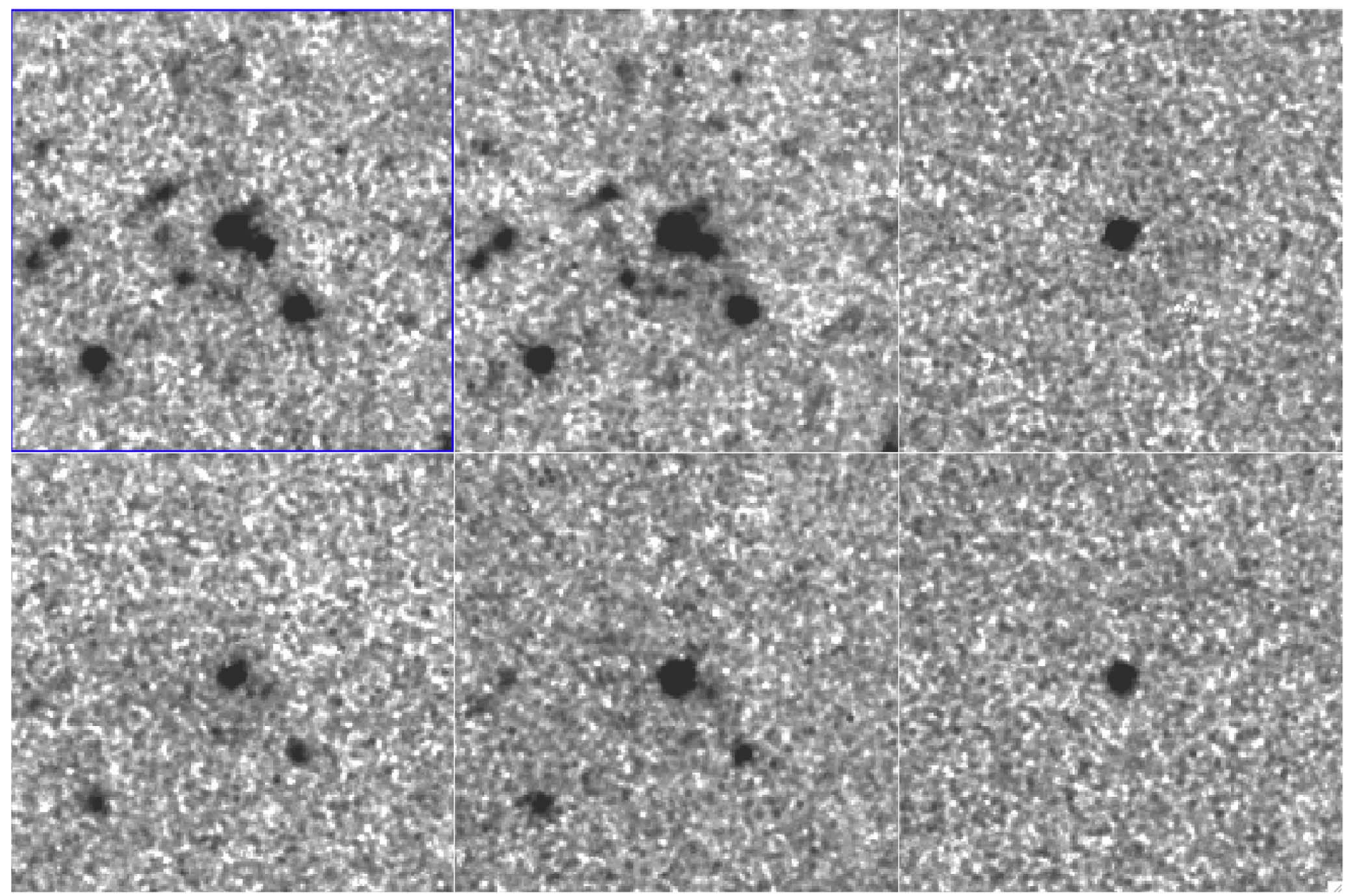

Figure 3. Top panels: montage of $W 1$ images showing a $180^{\prime \prime} \times 180^{\prime \prime}$ region centered on the position of $0145+234$ from the AllWISE Catalog. The left panel shows the coaddition of the 2014 January single exposures, the center panel is the coaddition of the 2019 January single exposures when the source was in outburst, and the right panel is the difference between the 2019 January and 2014 January images. Bottom panels: same as the top panels, but for $W 2$.

Table 1

Blackbody Model Fit to the MIR Excess

\begin{tabular}{|c|c|c|c|c|c|c|c|c|}
\hline \multirow{2}{*}{ Epoch } & \multirow{2}{*}{$\begin{array}{c}W 1 \\
\text { (mag) }\end{array}$} & \multirow{2}{*}{$\begin{array}{c}W 2 \\
\text { (mag) }\end{array}$} & \multicolumn{3}{|c|}{ Scenario $1^{\mathrm{a}}$} & \multicolumn{3}{|c|}{ Scenario $2^{\mathrm{b}}$} \\
\hline & & & $\begin{array}{c}T \\
(\mathrm{~K})\end{array}$ & $\begin{array}{c}A \\
\left(10^{16} \mathrm{~m}^{2}\right)\end{array}$ & $\begin{array}{c}R \\
(R)_{\odot}\end{array}$ & $\begin{array}{l}T \\
\mathrm{~K}\end{array}$ & $\begin{array}{c}A \\
10^{16} \mathrm{~m}^{2}\end{array}$ & $\begin{array}{c}R \\
R_{\odot}\end{array}$ \\
\hline $1-12$ & $13.87 \pm 0.04$ & $13.63 \pm 0.06$ & $1163 \pm 145$ & $3.54 \pm 1.50$ & $1.46 \pm 0.37$ & $\ldots$ & $\ldots$ & \\
\hline 13 & $12.94 \pm 0.01$ & $12.62 \pm 0.02$ & $1769 \pm 74$ & $4.80 \pm 0.51$ & $0.63 \pm 0.05$ & $1949 \pm 529$ & $2.99 \pm 1.88$ & $0.52 \pm 0.28$ \\
\hline 14 & $12.99 \pm 0.01$ & $12.60 \pm 0.02$ & $1515 \pm 62$ & $6.96 \pm 0.84$ & $0.88 \pm 0.07$ & $1570 \pm 329$ & $4.89 \pm 2.77$ & $0.80 \pm 0.34$ \\
\hline 15 & $12.79 \pm 0.02$ & $12.18 \pm 0.01$ & $1146 \pm 32$ & $23.06 \pm 2.08$ & $1.52 \pm 0.09$ & $1115 \pm 130$ & $21.34 \pm 8.80$ & $1.59 \pm 0.37$ \\
\hline
\end{tabular}

Notes.

${ }^{\mathrm{a}}$ Fitting excesses to the WD model.

${ }^{\mathrm{b}}$ Fitting quiescent state subtracted MIR flux.

is a new dust component that contributes to the brightening of the source. In the first case, we fit the total observed excess in the $W 1$ and $W 2$ bands at high state with a blackbody model. In the second case, we only fit the varying $W 1$ and $W 2$ fluxes with a blackbody model to constrain the newly formed dust component. The varying fluxes were calculated by subtraction of the mean quiescent flux from the high state fluxes, and uncertainties were calculated through propagations of errors. These results are summarized in Table 1 (see also Figure 5). In the first case, the temperature was increased from $1163 \mathrm{~K}$ to $1769 \mathrm{~K}$ from quiescent epochs (1-12) to epoch 13, while the surface area might be increased but the large uncertainties in the quiescent state make this inconclusive. In the subsequent epochs, the dust temperature is decreased continuously to
$1146 \mathrm{~K}$ at the last epoch, while the surface area increases by a factor of about four. In the second case, the newly formed dust had an initial temperature of about $1950 \mathrm{~K}$, and decreased to $1115 \mathrm{~K}$ at the last epoch, while surface area increased by a factor of about six.

With the above fit parameters, we can estimate physical quantities of the dust. The distance to the WD is $29.458 \pm$ $0.044 \mathrm{pc}$ using the parallax from the Gaia DR2 data (Gaia Collaboration et al. 2016, 2018; Lindegren et al. 2018), which is consistent with that derived from the SED fitting. We adopted the bolometric luminosity of $0.0035 L_{\odot}$ and WD radius of $0.0116 R_{\odot}$ from the spectroscopic WD model ${ }^{16}$ (Fontaine et al. 2001;

\footnotetext{
${ }^{16}$ http://montrealwhitedwarfdatabase.org/evolution.html
} 


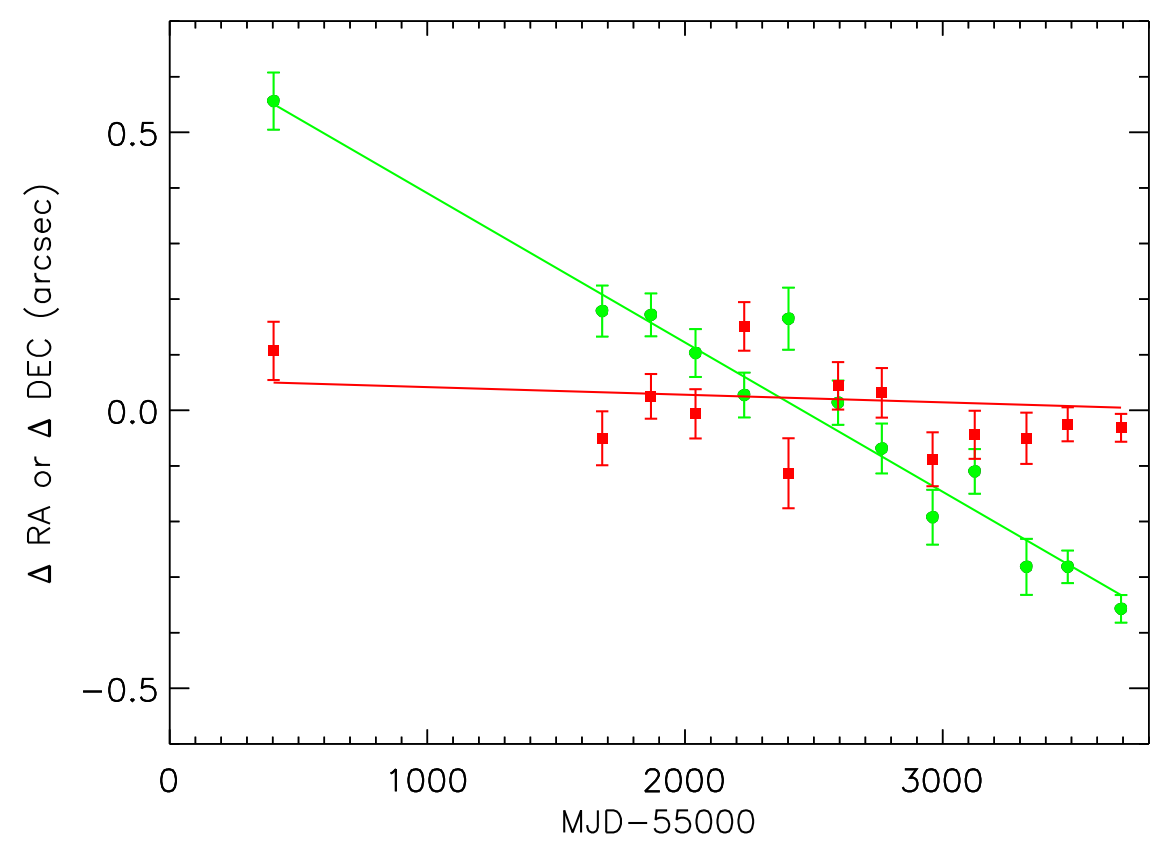

Figure 4. Relative R.A. (in red) and decl. (in green) motion of the MIR source with respect to their mean values during the WISE/NEOWISE surveys. The earliest points are from the AllWISE Catalog and represent the average positions from the 2010 January, 2010 July, and 2011 January measurement epochs. The points following MJD $=55600$ are the average positions measured during each six-month NEOWISE observation epoch. For comparison, the solid red and green lines show the expected R.A. and decl. motion of the white dwarf based on the Gaia astrometry and proper motion.

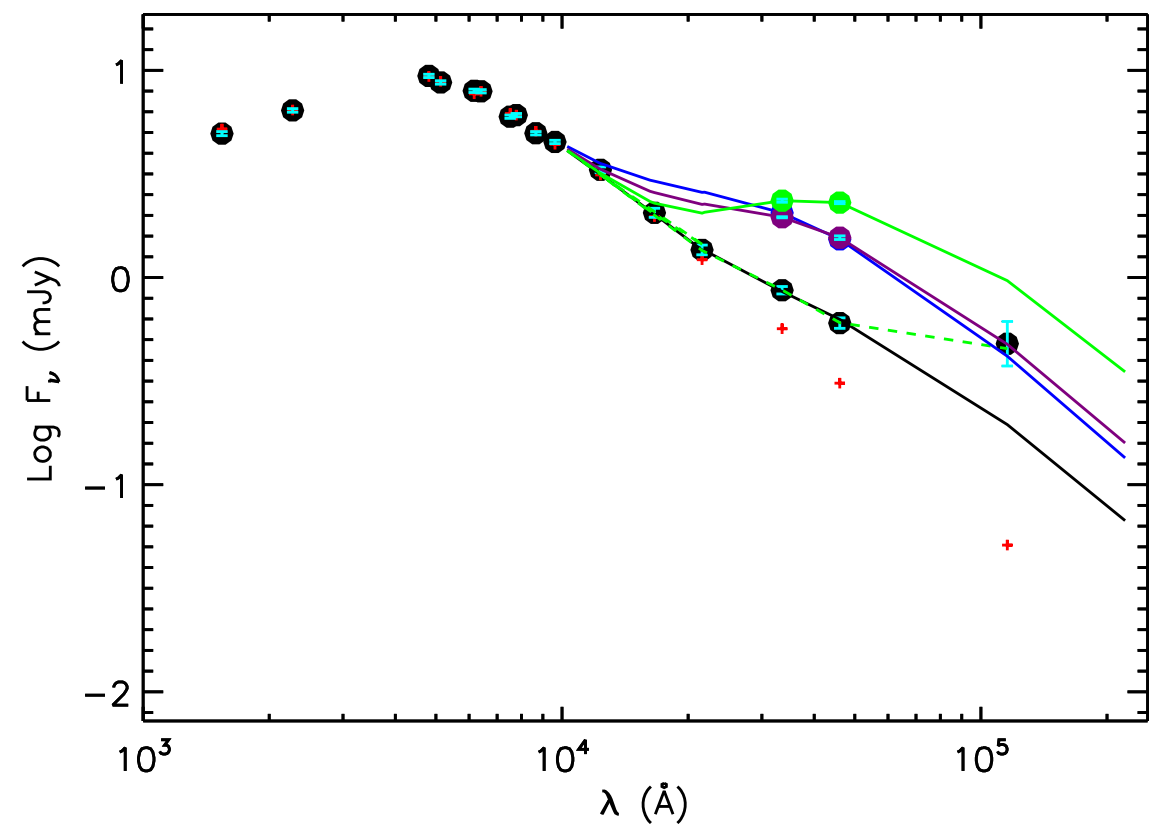

Figure 5. Spectral energy distribution (circles with error bars in cyan) and best-fitted models (cross or solid curve). The black, blue, purple, and green symbols represent the observed data on epochs $1-12,13,14$, and 15 in the light curve, respectively (see Table 1). The WD photosphere model is represented by red crosses. The solid curves represent the blackbody fit to the excesses over the WD photosphere model (colors follow the symbols). The PCA dust model is shown as the dashed green curve.

Gianninas et al. 2011). Assuming the dust is gray and in thermal equilibrium, we estimated the distance to the central star is on the order of

$$
R_{\mathrm{dust}}=\left(\frac{L_{\mathrm{WD}}}{4 \pi \sigma T_{d}^{4}}\right)^{1 / 2}=\left(T_{\mathrm{eff}} / T_{d}\right)^{2} R_{\mathrm{WD}}
$$

The distances of the dust to the WD are also listed in Table 1. The closest distance to the WD is about $0.5 R_{\odot}$.
The covering factor of the dust can be estimated from the ratio of the blackbody luminosity to the WD luminosity assuming dust is optically thick to the UV to optical light of the WD. The covering factor in the high state sharply rises to around $(1.5-2.0) \times 10^{-2}$ from $(2.7 \pm 0.3) \times 10^{-3}$ in the quiescent state, and remains nearly constant in the last three epochs.

Real dust is likely not gray, so the derived temperature and dust mass depends on the size distribution of grains $(N(a))$ and 

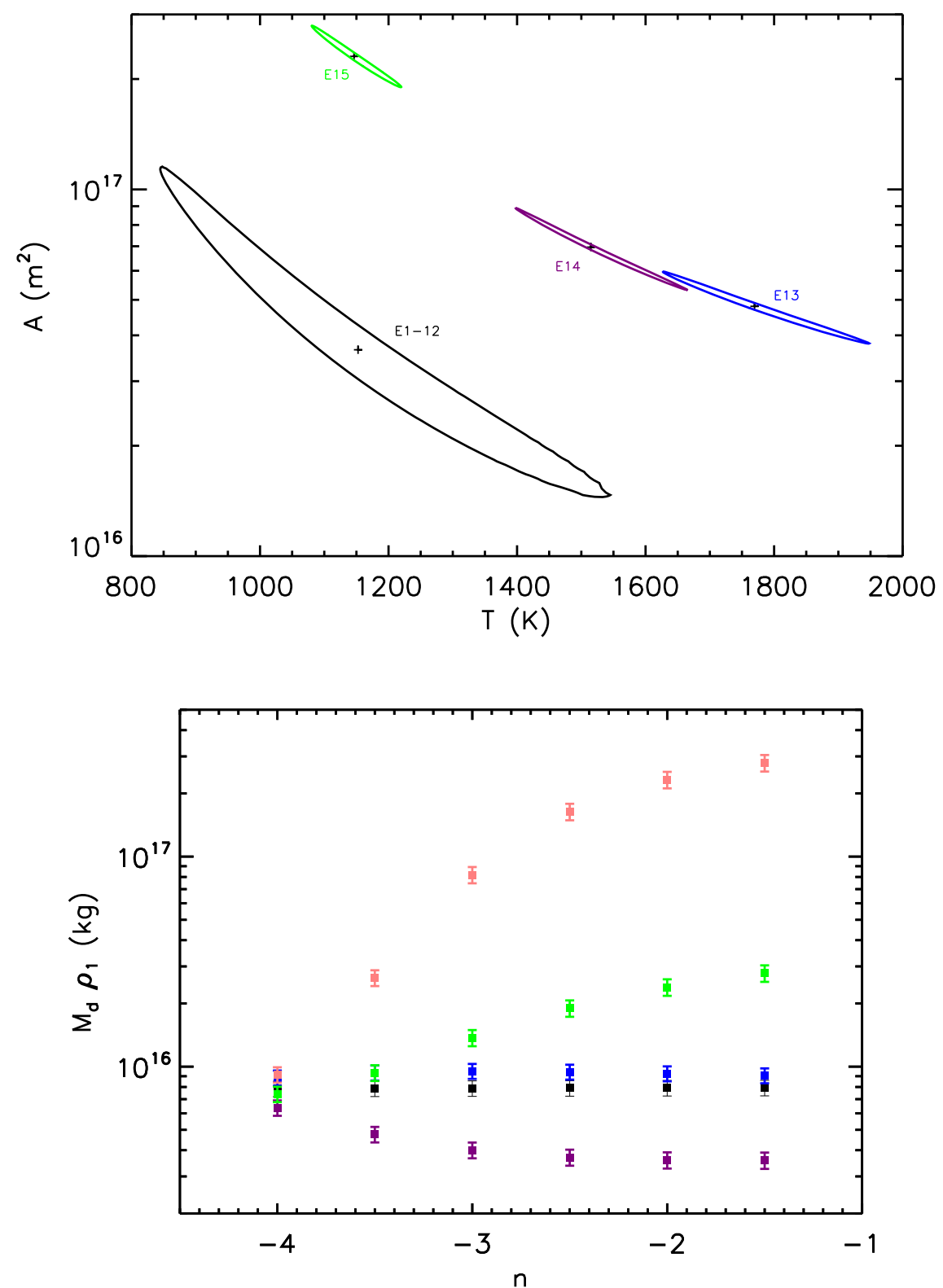

Figure 6. Upper panel: $90 \%$ contours for the blackbody fit to the excess IR flux on four epochs. The best-fitted parameters are marked as crosses. Colors are the same as those in Figure 5. Lower panel: the fitted dust mass of silicate grains as a function of power-law index at different cutoff of the largest grain size (red: 1000, green: 100, blue: 10 , black: 1 , and purple: $0.1 \mu \mathrm{m}$ ) for the last epoch of the NEOWISE-R survey.

their absorption coefficients $(Q)$. In the following, we assume that the sizes of dust grains follow a power-law distribution with an index $n$ in the range from $a_{\min }$ to $a_{\max }$ and none outside the range. The dust emission flux can be estimated

$$
\begin{aligned}
f_{\nu} & =\int_{a_{\min }}^{a_{\max }} N(a) 4 \pi a^{2} Q_{\nu}(a) d a \pi B_{\nu}(T) /\left(4 \pi d_{L}^{2}\right) \\
& =\frac{3 M_{d}}{4 \rho d_{L}^{2}}\left\langle\frac{Q_{\nu}}{a}\right\rangle B_{\nu}(T),
\end{aligned}
$$

where

$$
\left\langle\frac{Q_{\nu}}{a}\right\rangle=\frac{\int_{a_{\min }}^{a_{\max }} N(a) a^{3}(Q / a) d a}{\int_{a_{\min }}^{a_{\max }} N(a) a^{3} d a},
$$

and $M_{d}, \rho$ are the mass and density of the dust, respectively. The $d_{L}$ is the distance to the WD. In the blackbody case, the absorption coefficient is $Q_{\nu}=1$.
Using silicate coefficients ${ }^{17}$ in Laor \& Draine (1993), we calculated a set of $\left\langle\frac{Q_{\nu}}{a}\right\rangle$ for $a_{\min }=0.01 \mu \mathrm{m}$ and $a_{\max }=0.1,1$, $10,100,1000 \mu \mathrm{m}$. The excess infrared emission is then fitted with each of above models to derive mass and temperature of the dust. The lowest derived-temperatures are $T_{d}=770,1031$, $948,800 \mathrm{~K}$ for epochs $1-12,13,14,15$, respectively, or $60 \%$ $70 \%$ of those from the blackbody model, when $n=-4$ and $a_{\max }=0.1 \mu \mathrm{m}$. The highest temperatures are within $99 \%$ of those from the blackbody model when $n=-1$ and $a_{\max }=$ $1000 \mu \mathrm{m}$. The mass of dust is in the range of $3 \times 10^{15} \rho_{1}-3 \times$ $10^{17} \rho_{1} \mathrm{~kg}\left(\rho_{1}=\rho /\left(1 \mathrm{~g} \mathrm{~cm}^{-3}\right)\right)$, depending strongly on the size distribution (see Figure 6). A strict lower limit on the dust mass is a few times $10^{15} \mathrm{~kg}$, obtained when most grains have a size of about $1 \mu \mathrm{m}$, which gives the maximum $Q_{\nu} / a$ in the $W 1$ and

\footnotetext{
$\overline{17}$ For grains of size larger than $10 \mu \mathrm{m}$, we set $Q_{\lambda} \simeq 1$ for radiation at wavelengths greater than $4 \mu \mathrm{m}$.
} 
W2 bands. This minimum dust mass is very close to these of disks in the dustiest WD, e.g., $10^{15} \mathrm{~kg}$ in G29-38 (Reach et al. 2005 ) and $(0.3-1) \times 10^{15} \mathrm{~kg}$ in GD 362 (Jura et al. 2007). For a given distribution, dust mass increases monotonically with time. The increase of the amount of dust can be produced by further breakup of large bodies due to collision.

\section{Discussion}

The infrared light curve consists of a stable pre-burst quiescent phase, a short time rising outburst within half a year, and the MIR excess increasing phase with little variations in the optical light curve during the same period. This suggests that there is either a rapid inflow of an initial dust disk seen in the quiescent state into the inner region or tidal breakup of an asteroid into a clump of grains.

In order to understand various processes that may contribute to the dust changes, we examined the timescales of various processes, for the distance of dust to the host star of $\sim 1 R_{\odot}$, the WD mass of $0.667 M_{\odot}$, and the luminosity of $0.0035 L_{\odot}$. First, the dynamic time, or the orbit period, is about half an hour. Because we do not have the detailed light curve through the rising phase, we cannot reject the scenario that the newly formed dust is being brought by a giant comet. However, the late cooling (on a timescale of years) is too slow for a departing comet. It is highly unlikely that the event was caused by a passing comet.

Grains spiral onto the host star due to radiation drag (the Poynting-Robertson drag) on a timescale of (Burn 1979; Backman \& Paresce 1993)

$$
\begin{aligned}
t_{\mathrm{PR}}= & 4.73\left(\frac{b}{1 \mu m}\right)\left(\frac{\rho}{\mathrm{g} \mathrm{cm}^{-3}}\right)^{2}\left(\frac{R}{R_{\odot}}\right)^{2} \\
& \times\left(\frac{0.0035 L_{\odot}}{L_{W D}}\right) \frac{1}{1+\text { albedo }} \mathrm{yr},
\end{aligned}
$$

i.e., a few years in this case. Destruction of a large grain into small ones through collisions takes place on a timescale of (Backman \& Paresce 1993)

$$
t_{\mathrm{col}}=3.5\left(\frac{R}{R_{\odot}}\right)^{3 / 2}\left(\frac{0.667 M_{\odot}}{M_{\mathrm{WD}}}\right)^{1 / 2}\left(\frac{10^{-5} L_{\odot}}{L_{\mathrm{IR}}}\right) \mathrm{yr},
$$

which is long, $\sim 10 \mathrm{yr}$ for the quiescent dust disk. Accretion of dust due to friction in the dust disk is much longer than $10^{4} \mathrm{yr}$ (Rafikov 2011; Girven et al. 2012). Given that this outburst event timescale appears to be consistent with the radiation drag and collision dust destruction timescales, it is most likely that the dust is produced by tidal breakup of an asteroid-like object.

The MIR observations are consistent with this picture. The temperature in the initial rising phase is as high as $1770 \mathrm{~K}$ in the blackbody fit. Assuming the dust is in radiative equilibrium, we estimate the distance of the dust to the WD is about $(0.63 \pm 0.05) R_{\odot}$, which is consistent within a tidal disruption radius, $r_{t}=0.69\left(\frac{M_{\mathrm{WD}}}{0.667 M_{\odot}} \frac{3 \mathrm{~g} \mathrm{~cm}^{-3}}{\rho}\right)^{1 / 3} R_{\odot}$ of a gravitationally bounded asteroid with a typical density of $\rho=(1-7) \mathrm{g} \mathrm{cm}^{-3}$ (Carry 2012). The disrupted debris will have a range of specific energies and will be spread in space. A small fraction with the lowest energies may fall directly onto the $\mathrm{WD}$, and pollute the surface of the WD. A large part of debris may form an eccentric disk. Collision in the clump of debris will further cause fragmentation, releasing smaller grains, and thus increases the surface area of dust as observed. Similar events were detected before, for instance, evidence for planetesimals in an orbit close to the tidal disruption radius was reported in WD $1145+017$ through the transit signal (Vanderburg et al. 2015; Xu et al. 2016). More recently, Manser et al. (2019) reported detection of a planetesimal orbiting within the debris disk through its perturbation to the gas disk. Farihi et al. (2018) proposed that dust production and depletion through collisions (secular evolution) are common based on the detection of MIR variability of $20 \%$ peak-to-peak amplitude over $11 \mathrm{yr}$ in GD 56. Their light curve looked rather smooth. Xu et al. (2018) explored three mechanisms, including tidal disruption events, external perturbation, and runaway accretion, but did not reach a conclusion. The abrupt change in our object, on the other hand, suggests a catastrophic event. Our discovery may be the first case of an asteroid breakup process caught in action.

\section{Conclusion and Perspective}

We report a large MIR outburst of the WD 0145+234, which is still rising in the WISE W2 band. We interpret this event as tidal disruption of an asteroid by the WD. As the source MIR flux is still rising, further monitoring of this event will likely help provide insights to the fate of planet systems at their end of star lives. High resolution spectroscopic observations in the optical and UV can trace the composition of recent falling debris (asteroid; e.g., Swan et al. 2019b). According to the calculation given by Fontaine,${ }^{18}$ the diffusion time for most metals are less than a month. The disruption may also release volatile gas into the interplanetary space, leaving absorption lines in the UV and optical spectra (Wilson et al. 2019). The debris may contain warm gas emitting infrared Ca II emission lines, which can be used to trace the kinematic motion of the debris. Infrared photometric observations at long wavelengths and MIR spectroscopy can be also used to constrain dust composition.

We thank the referee for constructive comments. This work is supported by Chinese Science Foundation (NSFC-11833007, 11421303). This research makes use of data products from the Wide-field Infrared Survey Explorer, which is a joint project of the University of California, Los Angeles, and the Jet Propulsion Laboratory/California Institute of Technology, funded by the National Aeronautics and Space Administration. This research also makes use of data products from NEOWISE-R, which is a project of the Jet Propulsion Laboratory/California Institute of Technology, funded by the Planetary Science Division of the National Aeronautics and Space Administration. This research has made use of the NASA/ IPAC Infrared Science Archive, which is funded by the National Aeronautics and Space Administration and operated by the California Institute of Technology.

\section{ORCID iDs}

Ting-gui Wang (i) https://orcid.org/0000-0002-1517-6792 Ning Jiang (1) https://orcid.org/0000-0002-7152-3621 Roc M. Cutri (1) https://orcid.org/0000-0002-0077-2305 Peng Jiang (1) https://orcid.org/0000-0002-5387-7952 Zhengfeng Sheng (1) https://orcid.org/0000-0001-6938-8670 Hongyan Zhou (1) https://orcid.org/0000-0003-1956-9021 Edward L. Wright (1) https://orcid.org/0000-0001-5058-1593

\footnotetext{
18 http://montrealwhitedwarfdatabase.org/evolution.html
} 


\section{References}

Alexander, K. D., Berger, E., Guillochon, J., Zauderer, B. A., \& Williams, P. K. G. 2016, ApJL, 819, L25

Backman, D. E., \& Paresce, F. 1993, in Protostars and Planets III, ed. E. H. Levy \& J. I. Lunine (Tucson, AZ: Univ. Arizona Press), 1253

Bergeron, P., Rolland, B., Limoges, M.-M., et al. 2015, in ASP Conf. Ser. 493, 19th European Workshop on White Dwarfs, ed. P. Dufour et al. (San Francisco, CA: ASP), 33

Bergeron, P., Wesemael, F., Dufour, P., et al. 2011, ApJ, 737, 28

Blouin, S., Dufour, P., \& Allard, N. F. 2018, ApJ, 863, 184

Burn, I. 1979, JMatS, 14, 2453

Cardelli, J. A., Clayton, G. C., \& Mathis, J. S. 1989, ApJ, 345, 245

Carry, B. 2012, P\&SS, 73, 98

Chambers, K. C., Magnier, E. A., Metcalfe, N., et al. 2016, arXiv:1612.05560

Drake, A. J., Djorgovski, S. G., Mahabal, A., et al. 2009, ApJ, 696, 870

Farihi, J., Jura, M., \& Zuckerman, B. 2009, ApJ, 694, 805

Farihi, J., van Lieshout, R., Cauley, P. W., et al. 2018, MNRAS, 481, 2601

Fontaine, G., Brassard, P., \& Bergeron, P. 2001, PASP, 113, 409

Gaia Collaboration, Brown, A. G. A., Vallenari, A., et al. 2016, A\&A, 595, A2

Gaia Collaboration, Brown, A. G. A., Vallenari, A., et al. 2018, A\&A, 616, A1

Gänsicke, B. T., Marsh, T. R., Southworth, J., et al. 2006, Sci, 314, 1908

Gianninas, A., Bergeron, P., \& Ruiz, M. T. 2011, ApJ, 743, 138

Girven, J., Brinkworth, C. S., Farihi, J., et al. 2012, ApJ, 749, 154

Jura, M. 2003, ApJL, 584, L91

Jura, M., Farihi, J., \& Zuckerman, B. 2007, AJ, 133, 1927

Jura, M., \& Young, E. D. 2014, AREPS, 42, 45
Kochanek, C. S., Shappee, B. J., Stanek, K. Z., et al. 2017, PASP, 129, 104502 Koester, D. 2009, A\&A, 498, 517

Koester, D., Provencal, J., \& Gänsicke, B. T. 2014, A\&A, 568, A118

Laor, A., \& Draine, B. T. 1993, ApJ, 402, 441

Limoges, M.-M., Bergeron, P., \& Lépine, S. 2015, ApJS, 219, 19

Lindegren, L., Hernández, J., Bombrun, A., et al. 2018, A\&A, 616, A2

Mainzer, A., Bauer, J., Cutri, R. M., et al. 2014, ApJ, 792, 30

Manser, C. J., Gänsicke, B. T., Eggl, S., et al. 2019, Sci, 364, 66

Nakamura, R. 1998, EP\&S, 50, 587

Rafikov, R. R. 2011, MNRAS, 416, L55

Reach, W. T., Kuchner, M. J., von Hippel, T., et al. 2005, ApJL, 635, L161

Rebassa-Mansergas, A., Solano, E., Xu, S., et al. 2019, MNRAS, 489, 3990

Shappee, B. J., Prieto, J. L., Grupe, D., et al. 2014, ApJ, 788, 48

Skrutskie, M. F., Cutri, R. M., Stiening, R., et al. 2006, AJ, 131, 1163

Swan, A., Farihi, J., Koester, D., et al. 2019a, MNRAS, 490, 202

Swan, A., Farihi, J., \& Wilson, T. G. 2019b, MNRAS, 484, L109

Tremblay, P.-E., Bergeron, P., \& Gianninas, A. 2011, ApJ, 730, 128

Vanderburg, A., Johnson, J. A., Rappaport, S., et al. 2015, Natur, 526, 546

Veras, D., Leinhardt, Z. M., Bonsor, A., et al. 2014, MNRAS, 445, 2244

Wills, D., \& Wills, B. J. 1974, MNRAS, 167, 79P

Wilson, D. J., Gänsicke, B. T., Koester, D., et al. 2019, MNRAS, 483, 2941

Wright, E. L., Eisenhardt, P. R. M., Mainzer, A. K., et al. 2010, AJ, 140, 1868

Xu, S., \& Jura, M. 2014, ApJL, 792, L39

Xu, S., Jura, M., Dufour, P., et al. 2016, ApJL, 816, L22

Xu, S., Su, K. Y. L., Rogers, L. K., et al. 2018, ApJ, 866, 108

Zuckerman, B., \& Becklin, E. E. 1987, Natur, 330, 138

Zuckerman, B., Melis, C., Klein, B., et al. 2010, ApJ, 722, 725 